\title{
Characterization and electromagnetic response of a $\phi$-shaped metamaterial
}

\author{
N.T. Tung ${ }^{1,2, a}$, J.W. Park ${ }^{2}$, V.T.T. Thuy ${ }^{2, \mathrm{~b}}$, P. Lievens ${ }^{1}$, Y.P. Lee ${ }^{2}$, and V.D. Lam ${ }^{3, c}$ \\ 1 Laboratory of Solid State Physics and Magnetism, Department of Physics and Astronomy, Katholieke Universiteit Leuven, \\ 3001 Leuven, Belgium \\ 2 Quantum Photonic Science Research Center and Department of Physics, Hanyang University, 133-791 Seoul, Korea \\ 3 Institute of Materials Science, Vietnamese Academy of Science and Technology, Vietnam
}

Received 14 February 2011 / Received in final form 27 April 2011

Published online 7 June 2011 - (c) EDP Sciences, Società Italiana di Fisica, Springer-Verlag 2011

\begin{abstract}
In this paper we experimentally and numerically study the left-handed behavior in a biplanar metamaterial operating at microwave frequency. The metamaterial consists of $\phi$-shaped metallic patterns on both sides of a dielectric spacer. The origin of negative refractive index is elucidated by performing the effective medium analysis. Additionally, the dependence of the left-handed transmission on geometric parameters, such as the periodicities, the shapes of metallic slabs and continuous wires, and the impact of spacer separation, is examined.
\end{abstract}

\section{Introduction}

Recently, the understanding of wave-matter interaction has been significantly extended by the appearance of a new class of materials, the so-called metamaterials [1]. Metamaterials are artificially-engineered materials, which are built up from many small periodically inhomogeneous inclusions to enact the unique macroscopic electromagnetic behavior, such as the negative refraction or the left-handed (LH) property [2]. The wave propagation in a negative refractive medium that is supposed to be achieved by simultaneously negative permeability and permittivity, was theoretically discussed first by Veselago [3]. With the recent development in micro and nano fabrication, the realization of negative refraction has been experimentally confirmed at both microwave and optical frequencies with the great promise of advanced applications [4]. From the original proposal for microwave metamaterials using conducting wires providing negative permittivity [5] and splitring resonator (SRR) for negative permeability [6], most of the attention has been paid for searching a potential design with low loss and high advantages in fabrication technology [7-9].

In fact, the combination of SRRs and continuous wires has not been successful for negative refractive index in the optical range due to complications in practical implementations. A transformed structure from the symmetric SRR, hence, was proposed as a potential candidate for

\footnotetext{
a e-mail: thanhtung.nguyen@fys.kuleuven.be

b Present address: Institute of Physics, Vietnam Academy of Science and Technology, Hanoi, Vietnam.

c e-mail: lamvd@ims.vast.ac.vn
}

the negative permeability at both microwave and optical frequencies $[10,11]$. This design, named as cut-wire pairs (CWPs), with an addition of the continuous wires, allows us to obtain a strong LH behavior even with only a onelayer structure instead of stacking many layers to cover the incident electromagnetic wave. Most recently, the fishnet structure, which is obtained by broadening the width of CWPs to connect with the continuous wires, has been fabricated and shown to be a candidate for 3D optical negative refraction [12]. However, the large fraction of metal in fishnet metamaterials is an important drawback since it might lead to considerable losses and thus to saturation of the magnetic resonance frequency, especially in the optical domain [13].

In this paper, we report an observation of the LH behavior in a metamaterial structure at $\mathrm{GHz}$ frequencies. This structure, as a distinct transformation of the regular fishnet, is composed of a pair of metallic $\phi$-shaped patterns separated by a dielectric spacer [14]. The $\phi$-shaped structure was supposed to result in a better optical LH behavior than the fishnet one since it can exceptionally reduce the contribution of metallic losses. Additionally, this structure could provide more degrees of freedom for independently controlling the electric and the magnetic responses for a highly LH transmission by impedance matching [15]. Notwithstanding, the electromagnetic responses of the $\phi$ shaped structure have not been studied experimentally yet [14]. The aim of our efforts in the present study is to bridge this gap by investigating the characteristics of electromagnetic behavior of $\phi$-shaped structure. The nature of negative permeability and negative permittivity are experimentally and numerically clarified by effective medium 

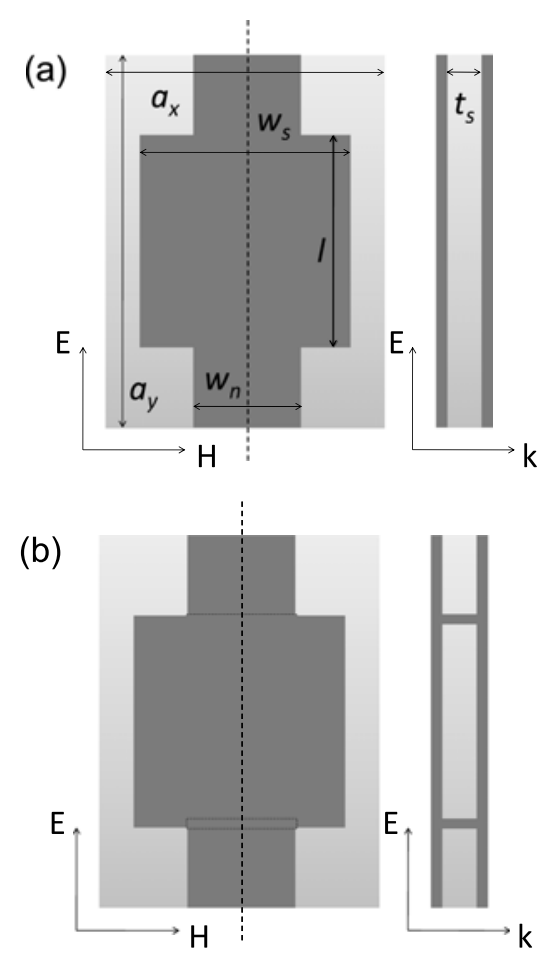

Fig. 1. (Color online) Schematic representing of the unit cell of (a) $\phi$-shaped metamaterials and (b) its magneticallyeliminated version with geometric parameters.

analysis. The effects of various geometrical parameters on the LH behavior of $\phi$-shaped structure are also discussed.

\section{Experimental procedure and numerical simulation}

A single unit cell of the $\phi$-shaped structure with the geometric parameters is shown in Figure 1. In both simulation and measurement, the incident wave was normal to the plane of structure, and the electric and the magnetic fields are parallel to the $y$ - and the $x$-axis, respectively. For the experiment, the $\phi$-shaped structure was fabricated by using the conventional printed circuit board process with $36 \mu \mathrm{m}$-thick copper coating on both sides of a FR4 dielectric spacer (see Fig. 2a). The $\phi$-shaped patterns are formed by masking and etching procedures. The length $\left(l_{s}\right)$ and the width $\left(w_{s}\right)$ of the metallic slab are 5.6 and $3.2 \mathrm{~mm}$, respectively, while the width of the continuous wires is $w_{n}=0.8 \mathrm{~mm}$. The periodicities $a_{x}$ and $a_{y}$ are 4.0 and $7.6 \mathrm{~mm}$, respectively. We performed the transmission measurements in free space at room temperature using a Hewlett-Packard E8362B network analyzer connected to the microwave standard gain horn antennas (see Fig. 2b).

For the numerical study, we employed the transfermatrix method (TMM) to calculate the transmission of the $\phi$-shaped structure. The original TMM method has been described in detail by Pendry et al. based on the scattering formalism to numerically reassert the scaling theory of localization [16-18]. In the TMM, the sample
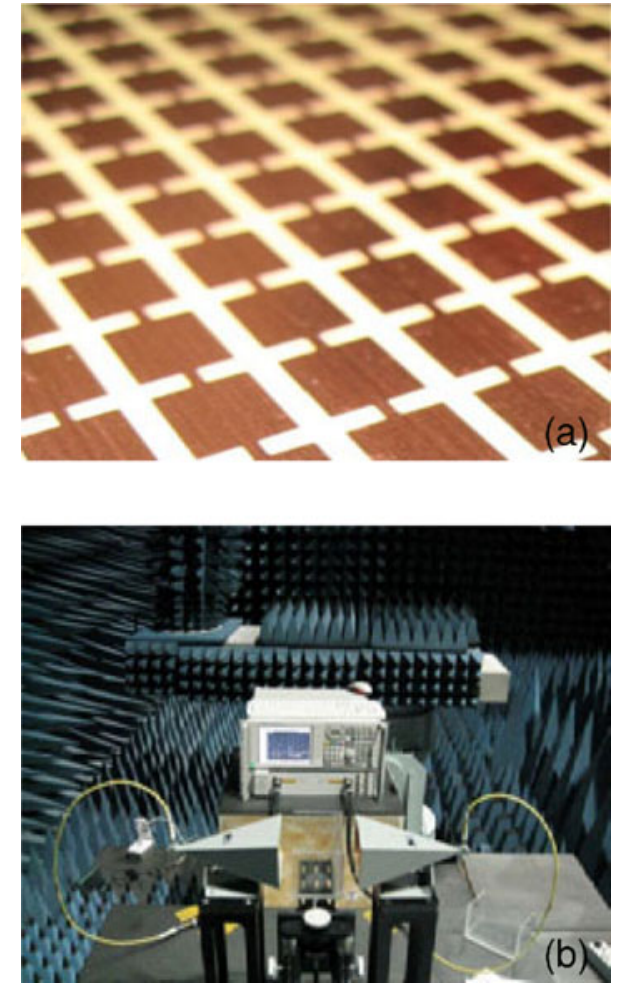

Fig. 2. (Color online) (a) Photograph of the actual $\phi$-shaped metamaterial structure. (b) Photo of the experimental setup for the transmission measurement.

is considered as a scatterer for the incoming wave and is characterized by four parameters: transmission of the wave from left to right $\left(t_{+}\right)$, from right to left $\left(t_{-}\right)$, and the reflection from right to right $\left(r_{+}\right)$and left to left $\left(r_{-}\right)$. The corresponding scattering matrix $S$ becomes

$$
S=\left(\begin{array}{c}
t_{+} r_{+} \\
r_{-} t_{-}
\end{array}\right)
$$

which determines the amplitudes of outgoing wave $B$ and $C$ in terms of the amplitudes of incoming wave $A$ and $D$, namely,

$$
\left(\begin{array}{l}
C \\
B
\end{array}\right)=S\left(\begin{array}{l}
A \\
D
\end{array}\right)
$$

It can be rewritten into the transfer matrix form as

$$
\left(\begin{array}{l}
D \\
C
\end{array}\right)=T\left(\begin{array}{l}
B \\
A
\end{array}\right)
$$

where $T$ is the transfer matrix

$$
T=\left(\begin{array}{cc}
t_{-}^{-1} & -t_{-}^{-1} r_{-} \\
r_{+} t_{-}^{-1} & t_{+}-r_{+} t_{-}^{-1} r_{-}
\end{array}\right)
$$

which determines the outgoing electromagnetic fields on one side of the structure from the incident fields. For the multi-layer structures, then the total transfer-matrix of the whole system can be calculated by following the composition law to be

$$
T_{1 n}=T_{n} \ldots T_{1}
$$



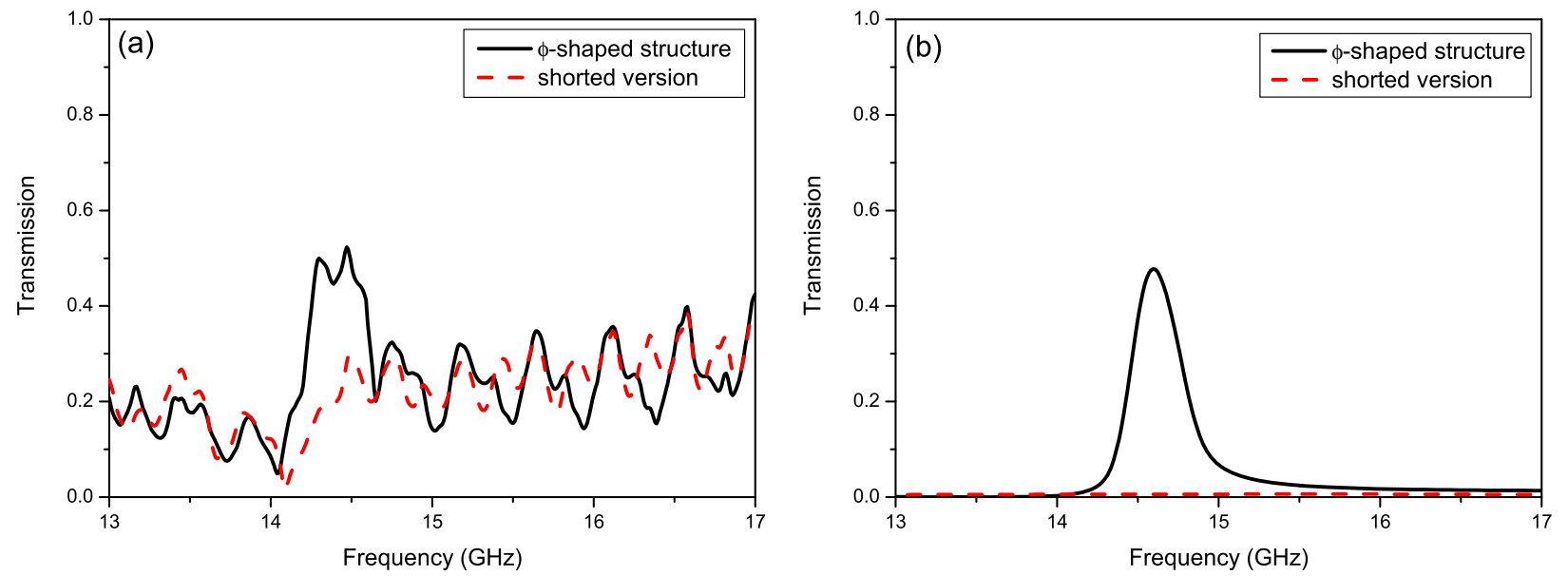

Fig. 3. (Color online) (a) Measured and (b) simulated transmission spectra of one-layer $\phi$-shaped structure and its shorted version. The thickness $t_{d}$ of the dielectric spacer is $0.4 \mathrm{~mm}$. Other parameters are given in Section 2 .

The total volume of the system is divided into $N_{x} \times N_{y} \times N_{z}$ mesh points by a discretization length $\delta$ so that each mesh point is regarded as a homogeneous medium. All length parameters are given as integer multiples of $\delta: a_{x}=\delta \times N_{x}, a_{y}=\delta \times N_{y}$ and $a_{z}=\delta \times N_{z}$. In our case, $\delta$ is $0.4 \mathrm{~mm}$ and $N_{x}=10, N_{y}=19$ and $N_{z}$ varies from 5 to 7 . There is no observable change in the outcome at smaller values of $\delta$. The dielectric constant of the FR4 spacer was kept equal to the practical value of 4.9. The metal used to form the $\phi$-shaped structure was assumed to be a perfect conductor, and the material properties are independent of the thickness in the frequency range of interest. Details about the optimization procedure have been reported elsewhere [16-18]. For each point of the lattice, four components of the electromagnetic field $E_{x}, E_{y}$, $H_{x}$ and $H_{y}$ are calculated. The fields in each cell are coupled to those in the neighboring ones. We assume periodic boundary conditions in the direction along the interfaces and the embedded reference medium is vacuum.

\section{$3 \mathrm{LH}$ transmission in $\phi$-shaped structure}

We begin by utilizing the measurements and the simulations to discuss the characterizations of the $\phi$-shaped design and the origin of its LH behavior. The measurements have been carried out by a medium of $30 \times 15 \times 1$ unit cells of $\phi$-shaped structure (with one unit cell along the propagation direction) in free space, providing full coverage for the incident beam. The simulated transmission has been calculated using the transfer-matrix method with periodic boundary conditions along the $\mathbf{E}$ - and the $\mathbf{H}$-directions.

The most fundamental way to qualitatively study the $\mathrm{LH}$ behavior of metamaterials is the usage of effective medium analysis [19]. Generally, the $\phi$-shaped structure can be achieved from the combined metamaterials [14] by horizontally expanding the width of paired slabs (or CWP structure) in one direction to connect with the continuous wires. The paired-slab structure has been used extensively as a negative permeability meta-atom while the periodic

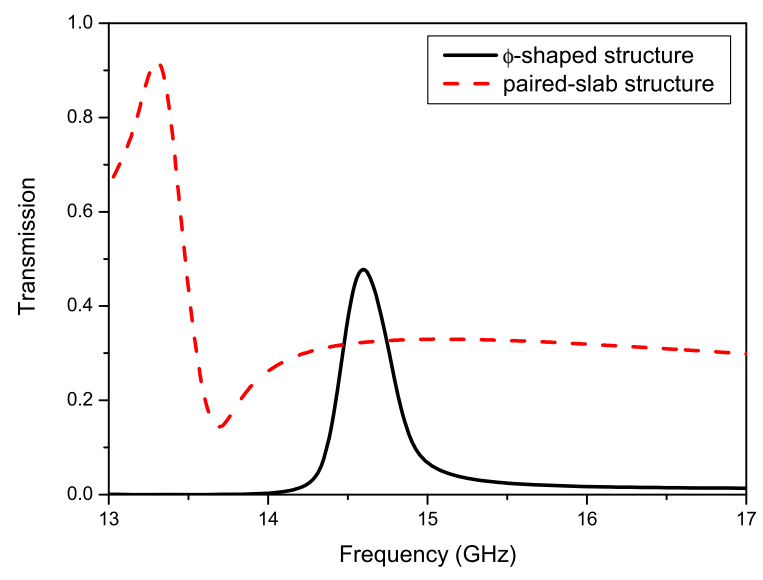

Fig. 4. (Color online) Simulated transmission spectra of $\phi$-shaped and paired-slab structure.

array of continuous wires has been demonstrated as a lowfrequency plasma provider. The negative permeability of the $\phi$-shaped structure is usually originated by the circular currents between each pair of slabs, which result in the induced magnetic dipole moments opposed to the applied field. Meanwhile, the electric response is inferred as the plasma behavior from continuous wires with an additional electric dipole from paired slabs. The LH transmission in a $\phi$-shaped structure, therefore, is assumed to occur when the magnetic resonance and the total plasma are coinciding. By comparing only the transmission data of magnetically and electrically eliminated structures with the original one, the magnetic, the electric and the $\mathrm{LH}$ responses of the given medium can be systematically determined.

The results of the effective medium analysis are shown in Figures 3 and 4. The measured and the simulated transmission spectra of the $\phi$-shaped structure and its magnetically eliminated version are plotted in Figures $3 \mathrm{a}$ and $3 \mathrm{~b}$ with an excellent agreement. The fluctuations in experimental data might be due to noise multi-scattered from the samples. The magnetically eliminated version is obtained by shortening the ends of slabs to remove the 

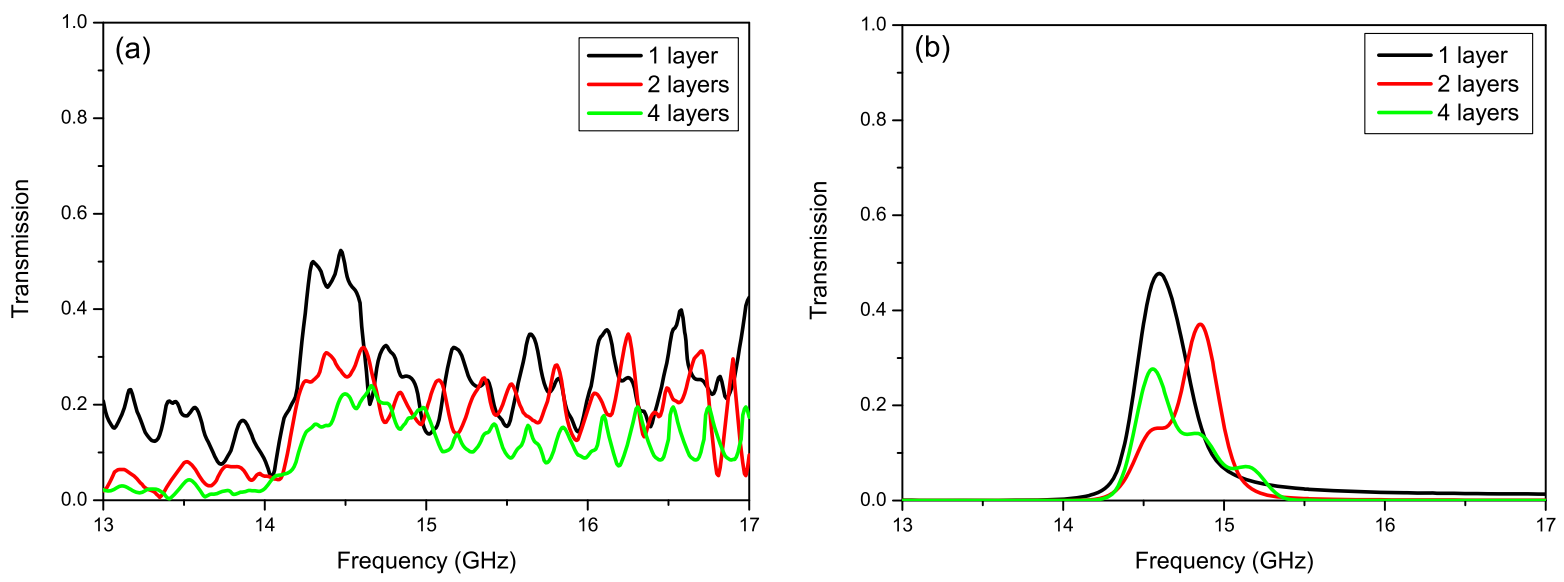

Fig. 5. (Color online) (a) Measured and (b) simulated transmission spectra of one-, two- and four-layer $\phi$-shaped structures. The distance between layers is kept to be $0.8 \mathrm{~mm}$.

effective capacitance, expecting a behavior as a sum of the electric-dipole CWs plus the plasma continuous wires. By this technique, the driving force in the circular currents is removed and, hence, the magnetically originated resonance in the $\phi$-shaped structure disappears without any significant effect on the electric response. As can be seen in Figure 3, there is a striking peak at around $14.5 \mathrm{GHz}$ in the transmission spectra of the $\phi$-shaped structure; however, it is entirely vanishing when the magnetic resonance is switched off in the shorted version. It indicates that the position of the transmission peak is clearly associated with the magnetic resonance of the $\phi$-shaped structure while the plasma behavior of the $\phi$-shaped structure is similar to that of continuous wires in the $13-17 \mathrm{GHz}$ range. Importantly, it is shown that the transmission peak at around 14.5 $\mathrm{GHz}$ is resulting from the simultaneously negative permeability and permittivity, in other words, the LH behavior.

In an effort to clarify the magnetic responses in case of the $\phi$-shaped and the CWP structures, we present their transmission spectra in Figure 4 . It is expected that the magnetic resonance of only CWPs should superpose with the LH peak of the $\phi$-shaped structure as aforementioned. Clearly, one can observe a transmission dip regarding the magnetic resonance in the CWP structure, whose frequency, nevertheless, is slightly lower than the LH peak of the $\phi$-shaped one. The possible reason is due to the additional inductance of the added metallic pattern after the CWPs widen to combine with the continuous wires, forming the $\phi$-shaped structure. Similar results were also observed in the fishnet structures $[14,20]$.

The LH performance for multi-layer systems of $\phi$-shaped structures is further examined in Figure 5. For this purpose, the transmission spectra of one-, two- and four-layer systems with a separation between layers of $0.8 \mathrm{~mm}$ are measured and compared with the simulations. A glass holder was used to keep samples properly aligned. It can be seen that the LH frequency of multi-layer systems is converging but the magnitude of the LH peak considerably decreases. This can be understood by increasing the loss in dielectric substrates. Additionally, it seems that the LH peak splits into several sub-resonances with increasing number of layers, which might be explained by the interaction between the patterns on the front side of one layer with the back side of the next one, and so on [21].

\section{Effects of geometric parameters}

In this section, we examine the geometric effects on the LH transmission of the $\phi$-shaped structure, which are helpful in optimizing the structure as well as simplifying the fabrication procedure. Qualitatively, the LC model of $\phi$-shaped structure can be described by the simple formula as follows:

$$
\omega_{m}^{2}=\omega_{m(\text { paired slabs })}^{2}+\frac{1}{L_{n} C}
$$

in which $\omega_{m}$ is the magnetic resonance frequency and $L_{n}$ is the inductance of added continuous wires [14]. Because of the magnetic resonance generated by the paired slabs, the change of geometric parameters in such structure should cause a considerable change on the LH properties of $\phi$-shaped structures. Therefore, in attempting to take into account the most complete effects of geometric parameters, we extend the study to the spacer separation dependence and the influences of lattice parameters on the LH transmission of the $\phi$-shaped structure.

In Figure 6 , the effect of spacer separation $t_{d}$, which has been neglected in the previous work [22], is experimentally and numerically studied in the present paper. In the hybridization scheme, the magnetic resonance of the $\phi$-shaped structure is considered as the antisymmetric energy mode of a paired-slab structure. With this picture in mind, one can imagine that increasing the spacer separation might result in a weaker attractive interaction, giving rise to a higher frequency of the antisymmetric energy mode and consequently, a higher frequency of the LH transmission of the $\phi$-shaped structure [23-25]. Part a of Figure 6 plots the measured and the simulated central frequencies of LH transmission of a one-layer $\phi$-shaped structure as function of the spacer separation $t_{d}$. The experiment and the simulation show a good agreement. It 

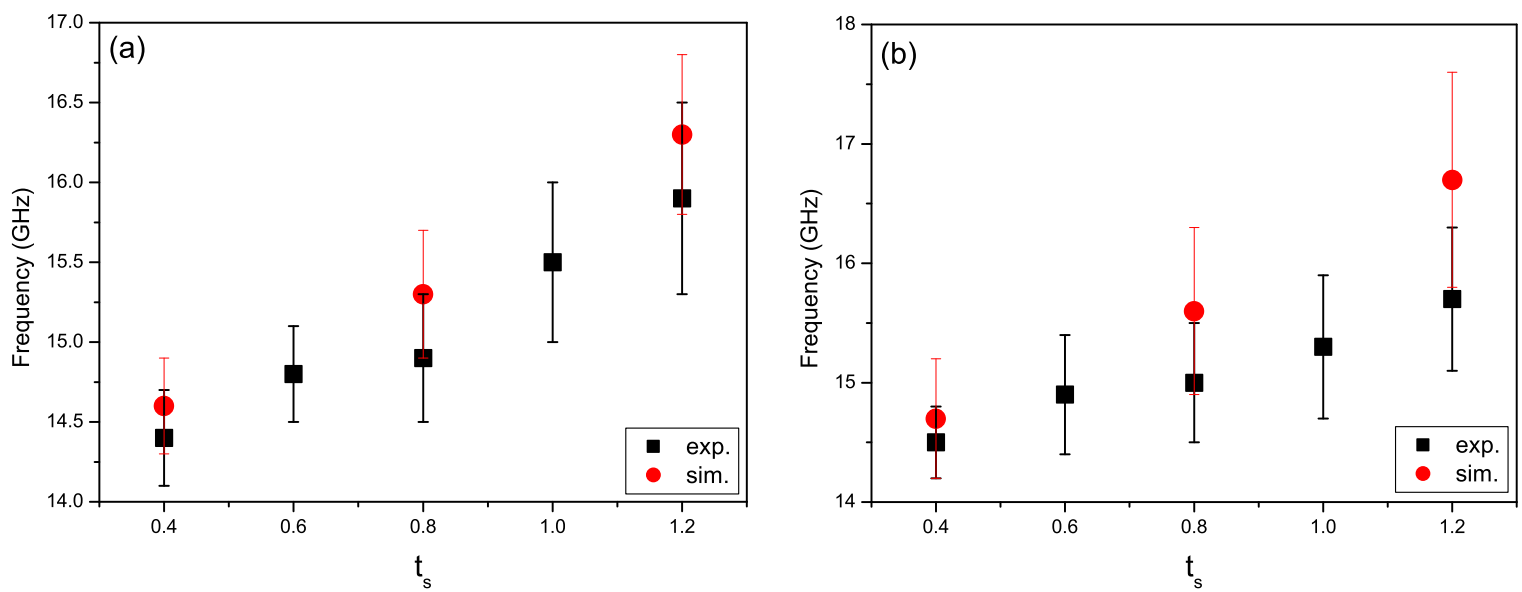

Fig. 6. (Color online) LH frequencies and their full widths at half maximum of (a) one- and (b) two-layer $\phi$-shaped structures as function of the spacer separation.

is observed that the central frequency of the LH peak linearly increases from 14.4 to $15.8 \mathrm{GHz}$ in the experimental data and from 14.6 to 16.3 in the simulated ones, with $t_{d}$ from 0.4 to $1.2 \mathrm{~mm}$. In part b of Figure 6, experiment and simulation for the two-layer $\phi$-shaped structure confirm the above argument. The distance between layers is kept as $0.8 \mathrm{~mm}$. As expected, the measured LH frequency increases from 14.5 to $15.7 \mathrm{GHz}$ while the simulated one changes from 14.6 to $16.6 \mathrm{GHz}$. The slight discrepancy between experiment and simulation can be understood by the increase of multi-scattering loss and the misalignment between layers in the microwave measurement.

In Figure 7, we present the simulated transmission spectra of the $\phi$-shaped structure according to the lattice constants $a_{x}$ (a) and $a_{y}$ (c) and according to the width $w_{s}$ (b) and the length $l_{s}(\mathrm{~d})$ of paired slabs. We begin by considering the influence of the distance between $\phi$-shaped structures along the $\mathbf{H}$ direction (as function of the lattice constant $a_{x}$ ) on the LH transmission. For this purpose, $a_{x}$ is varied from 3.6 to $4.8 \mathrm{~mm}$, while $a_{y}$ and $l_{s}$ are kept as 7.6 and $5.6 \mathrm{~mm}$, respectively. The transmission spectra of these structures are shown in Figure 7a. It is found that the LH frequency slightly increases from 14.4 to $14.8 \mathrm{GHz}$ with expanding the periodicity $a_{x}$ from 3.6 to $4.8 \mathrm{~mm}$. Another point is that the LH peak is somewhat intensified with larger $a_{x}$ value. Since the only varied parameter is the lattice constant $a_{x}$ which determines the distance between unit cells along the $\mathbf{H}$ direction, the shift apparently comes from the magnetic-coupling effect between neighboring unit cells when they progressively approach [26]. This effect induces a mutual inductance between two magnetic components and depends on the separation distance between the magnetic components. Similar results are observed in Figure $7 \mathrm{~b}$ with changing $w_{s}$; however, the LH frequency of the $\phi$-shaped structure is shown to increases more significantly with reducing $w_{s}$ than expanding $a_{x}$. The sensitivity to $w_{s}$ of the $\phi$-shaped structure might come from the magnetic resonance shift in paired slabs with changing width [14] rather than by the magnetic-coupling effect between neighboring unit cells, which has not been identified in fishnet structures. Another important advantage of the $\phi$-shaped structure is that its plasma frequency is pushed to the higher regime due to the smaller $w_{s}$ than that of fishnet structure $[14,20,27]$. Therefore, the LH peak could be well recognized practically without being blurred by a nearly right-handed one.

The dependence of magnetic resonance of paired slabs on the periodicity along the $\mathbf{E}$-direction and the length of slabs has been theoretically predicted by Zhou et al. In part (c) and (d) of Figure 7, we numerically verify this argument with our simulation method by presenting the transmission spectra of the $\phi$-shaped structure according to $a_{y}$ and $l_{s}$ ( $a_{x}$ and $w_{s}$ are kept at 4.0 and $3.2 \mathrm{~mm}$, respectively). As expected, the LH transmission is not considerably affected by $a_{y}$ while the LH frequency is clearly increased, in agreement with the analytic model. Note that the enhancement in magnitude of transmission for decreasing $l_{s}$ or increasing $w_{s}$ can be explained by the impedance matching of the structure [15].

\section{Conclusions}

We report the fabrication, experimental and numerical investigation of a $\mathrm{LH} \phi$-shaped metamaterials working at microwave frequency. The effective medium analysis through experiments and simulations was presented to interpret the titled LH behavior in such structure. The characterizations and the role of geometrical effects on such structure are also discussed. It is revealed that the $\phi$-shaped structure offers more widened parameters for optimization of LH implementations, such as the spacer separation and the width of paired slabs, than its analog, the fishnet structure. Additionally, this structure could exhibit a high LH performance due to the reduction of metallic loss especially at optical frequencies.

N.T. Tung would like to thank Dr. M.H. Cho at University of North Carolina for valuable discussions on the numerical method. This work is supported by the National Program for Basic researches in Natural Science of Vietnam (NAFOSTED) under contract No. 103.02.36.09 and the National Key 

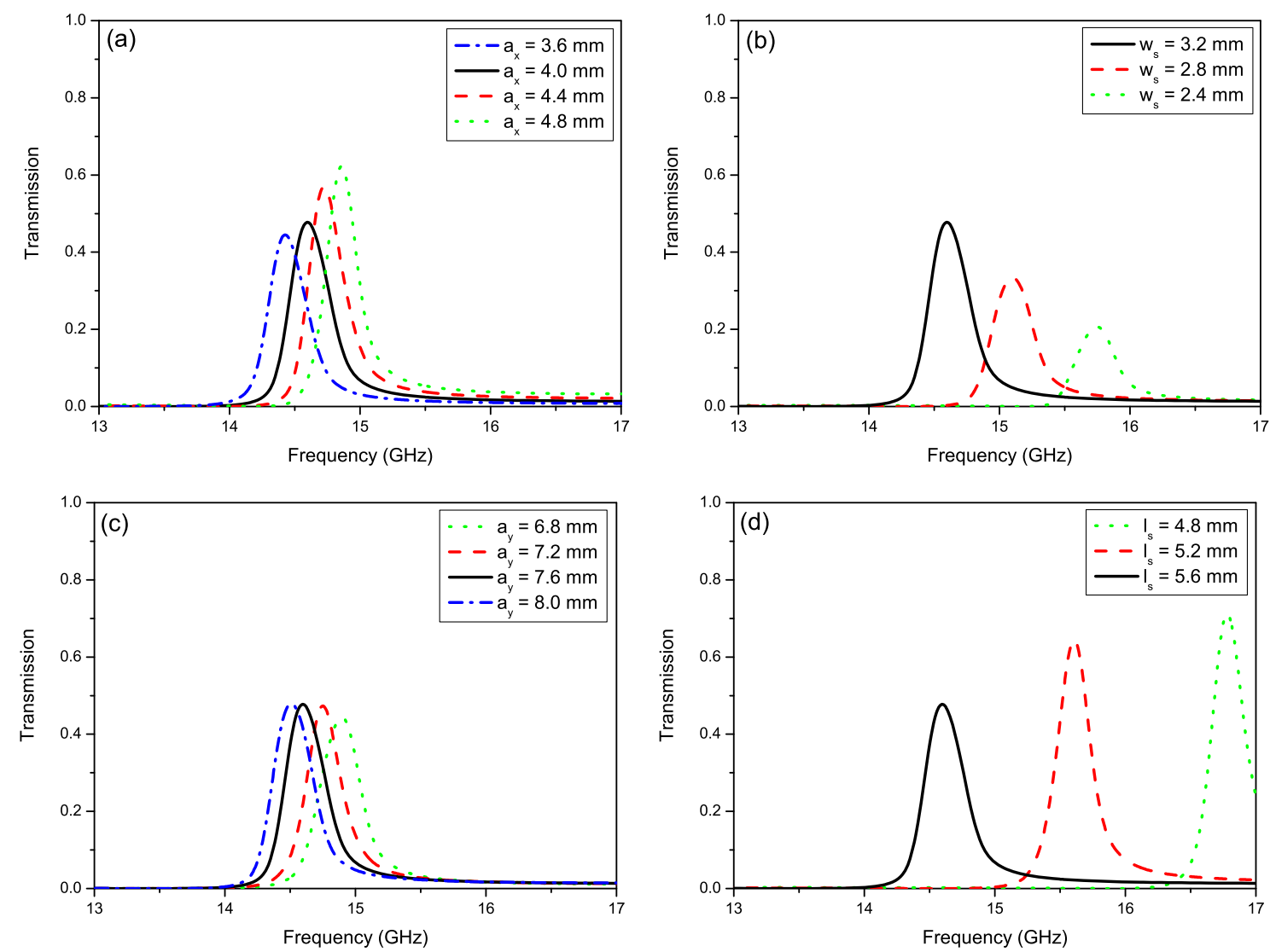

Fig. 7. (Color online) Simulated transmission spectra of $\phi$-shaped structure according to (a) $a_{x}$, (b) $w_{s}$, (c) $a_{y}$ and (d) $l_{s}$.

Laboratory in Electronic Materials and Devices, Institute of Materials Science, Vietnam Academy of Science and Technology, Vietnam.

\section{References}

1. J.B. Pendry, Contemp. Phys. 45, 191 (2004)

2. N. Engheta, R.W. Ziolkowski, Metamaterials: Physics and Engineering Explorations (Wiley, New York, 2006)

3. V.G. Veselago, Sov. Phys. Usp. 10, 509 (1968)

4. V.M. Shalaev, Nature Photonics 1, 41 (2007)

5. J.B. Pendry, A.J. Holden, W.J. Stewart, I. Youngs, Phys. Rev. Lett. 76, 4773 (1996)

6. J.B. Pendry, A.J. Holden, D.J. Robbin, W.J. Stewart, IEEE Trans. Microw. Theory Tech. 47, 2075 (1999)

7. H. Chen, L. Ran, J. Huangfu, X. Zhang, K. Chen, T.M. Grzegorczyk, J.A. Kong, Phys. Rev. E 70, 057605 (2004)

8. J. Huangfu, L. Ran, H. Chen, X.M. Zhang, K. Chen, T.M. Grzegorczyk, J.A. Kong, Appl. Phys. Lett. 84, 1537 (2004)

9. Z.G. Dong, S.Y. Lei, Q. Li, M.X. Xu, H. Liu, T. Li, F.M. Wang, S.N. Zhu, Phys. Rev. B 75, 075117 (2007)

10. V.M. Shalaev, W. Cai, U.K. Chettiar, H.K. Yuan, A.K. Sarychev, V.P. Drachev, A.V. Kildishev, Opt. Lett. 30, 3356 (2005)

11. G. Dolling, C. Enkrich, M. Wegener, C.M. Soukoulis, S. Linden, Science 312, 892 (2006)

12. J. Valentine, S. Zhang, T. Zentgraf, E.U. Avila, D.A. Genov, G. Bartal, X. Zhang, Nature (London) 455, 376 (2008)
13. J. Zhou, Th. Koschny, M. Kafesaki, E.N. Economou, J.B. Pendry, C.M. Soukoulis, Phys. Rev. Lett. 95, 223902 (2005)

14. M. Kafesaki, I. Tsiapa, N. Katsarakis, Th. Koschny, C.M. Soukoulis, E.N. Economou, Phys. Rev. B 75, 235114 (2007)

15. N.T. Tung, T.X. Hoai, V.D. Lam, J.W. Park, V.T. Thuy, Y.P. Lee, Eur. Phys. J. B 74, 47 (2010)

16. J.B. Pendry, A. MacKinnon, Phys. Rev. Lett. 69, 2772 (1992)

17. J.B. Pendry, J. Mod. Opt. 41, 209 (1994)

18. P. Markos, C.M. Soukoulis, Phys. Rev. B 65, 033401 (2001)

19. T. Koschny, M. Kafesaki, E.N. Economou, C.M. Soukoulis, Phys. Rev. Lett. 93, 107402 (2004)

20. V.D. Lam, J.B. Kim, S.J. Lee, Y.P. Lee, J. Appl. Phys. 103, 033107 (2008)

21. P. Gay-Balamz, O.J.F. Martin, J. Appl. Phys. 92, 2929 (2002)

22. J. Zhou, E.N. Economon, T. Koschny, C.M. Soukoulis, Opt. Lett. 31, 3620 (2006)

23. B. Kante, S.N. Burokur, A. Sellier, A. de Lustrac, J.-M. Lourtioz, Phys. Rev. B 79, 075121 (2009)

24. V.D. Lam, N.T. Tung, M.H. Cho, J.W. Park, W.H. Jang, Y.P. Lee, J. Phys. D Appl. Phys. 42, 115404 (2009)

25. N.T. Tung, V.D. Lam, M.H. Cho, J.W. Park, W.H. Jang, Y.P. Lee, Photonics Nanostruct. Fundam. Appl. 7, 206 (2009)

26. D.R. Smith, S. Schultz, P. Markos, C.M. Soukoulis, Phys. Rev. B 65, 195104 (2002)

27. K. Alici, E. Ozbay, J. Phys. D 41, 135011 (2008) 